\title{
Neuronal Population Codes and the Perception of Object Distance in Weakly Electric Fish
}

\author{
John E. Lewis and Leonard Maler \\ Department of Cellular and Molecular Medicine, University of Ottawa, Ottawa, Ontario, Canada K1H 8M5
}

\begin{abstract}
Weakly electric fish use an electric sense to navigate and capture prey in the dark. Objects in the surroundings of the fish produce distortions in their self-generated electric field; these distortions form a two-dimensional Gaussian-like electric image on the skin surface. To determine the distance of an object, the peak amplitude and width of its electric image must be estimated. These sensory features are encoded by a neuronal population in the early stages of the electrosensory pathway, but are not represented with classic bell-shaped neuronal tuning curves. In contrast, bell-shaped tuning curves do characterize the neuronal responses to the location of the electric image on the body surface, such that parallel two-dimensional maps of this feature are formed. In the case of such twodimensional maps, theoretical results suggest that the width of neural tuning should have no effect on the accuracy of a
\end{abstract}

In many sensory systems, neurons in the early processing stages are tuned to a specific two-dimensional (2D) location of a stimulus. In the visual system, this corresponds to the $2 \mathrm{D}$ projection of the visual world onto the retina; in the somatosensory system, this is the location of a touch on the skin. The neurons in these systems respond maximally for one location, with their activity decreasing for locations away from this preferred location; hence the neural responses are described by $2 \mathrm{D}$ bell-shaped tuning curves. Typically, these neurons have preferred locations distributed over a wide space such that a neural map of stimulus location is formed (Konishi, 1986; Knudsen et al., 1987). This is often referred to as a coarse code for stimulus location (Churchland and Sejnowski, 1992). Populations of neurons can also carry information about sensory features to which its component neurons are not explicitly tuned in this manner. In somatosensory processing, the $2 \mathrm{D}$ location of a skin probe is coarse-coded by peripheral mechanosensory neurons; yet humans cannot only determine the location of the probe, but can also accurately determine its shape, a feature that is not encoded with bellshaped tuning curves (Wheat et al., 1995; Khalsa et al., 1998). We refer to such population codes, which involve multiple coding strategies, as combined codes.

Weakly electric fish must use a combined population code during electrosensory processing. These fish can accurately determine the locations of objects in their surroundings using an

\footnotetext{
Received Sept. 22, 2000; revised Jan. 18, 2001; accepted Jan. 23, 2001.

This study was supported by the Canadian Institutes of Health Research through an operating grant to L.M. and a postdoctoral fellowship to J.E.L. Thanks to T. Lewis and S. Kealey for helpful comments on this manuscript.

Correspondence should be addressed to John E. Lewis, Department of Cellular and Molecular Medicine, University of Ottawa, 451 Smyth Road, Ottawa, Ontario, Canada K1H 8M5. E-mail: jlewis@uottawa.ca.

Copyright (C) 2001 Society for Neuroscience $0270-6474 / 01 / 212842-09 \$ 15.00 / 0$
}

population code. Here we show that although the spatial scale of the electrosensory maps does not affect the accuracy of encoding the body surface location of the electric image, maps with narrower tuning are better for estimating image width and those with wider tuning are better for estimating image amplitude. We quantitatively evaluate a two-step algorithm for distance perception involving the sequential estimation of peak amplitude and width of the electric image. This algorithm is best implemented by two neural maps with different tuning widths. These results suggest that multiple maps of sensory features may be specialized with different tuning widths, for encoding additional sensory features that are not explicitly mapped.

Key words: depth perception; electrolocation; electrosensory system; neuronal tuning; population coding; sensory coding active electric sense, a behavior called electrolocation (Heiligenberg, 1991; von der Emde et al., 1998). Objects with electrical properties that differ from those of the ambient water produce distortions in the fish's self-generated electric field. On the body surface, these distortions form a 2D electric image (Fig. 1) and provide the sensory input required to accurately encode object location in 3D (Rasnow, 1996; von der Emde et al., 1998). The electric image is initially encoded in the activity of skin electroreceptors. These receptors contact primary afferents that project somatotopically to the hindbrain and terminate in parallel on four maps in the electrosensory lateral line lobe (ELL). Each map is different in size and comprises pyramidal neurons with distinct physiological properties, including tuning curve width (Shumway, 1989a,b; Metzner, 1999; Turner and Maler, 1999). The necessary features of the electric image must be encoded in these $2 \mathrm{D}$ arrays of ELL pyramidal neurons. Object location in the 2D body plane is coarse-coded. Object distance (i.e., the third dimension) must be estimated indirectly from population activity related to the width and peak amplitude of the electric image (Rasnow, 1996; Assad et al., 1999).

Theoretical results suggest that the accuracy of a 2D coarse code should be unaffected by the width, and overlap, of the tuning curves (Snippe and Koenderink, 1992; Abbott and Dayan, 1999; Zhang and Sejnowski, 1999). Nonetheless, multiple parallel maps, exhibiting neuronal tuning with different widths and extents of overlap, are universal in sensory systems, even when they do not exist at the sensory periphery (Konishi, 1986). Different maps may provide multiple samples of information that can be averaged by downstream networks for higher accuracy. Alternatively, the different maps may be optimized to encode additional stimulus features using other strategies. The different ELL maps appear to be specialized; in some situations, information from each map is 
a

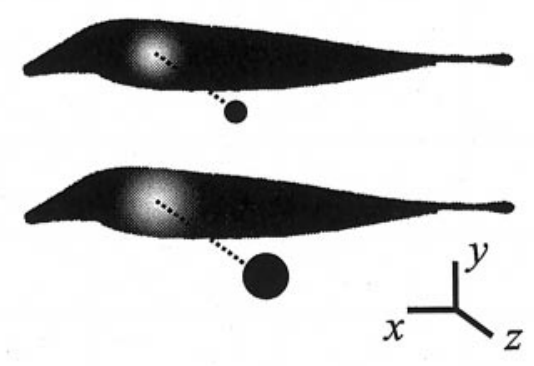

b

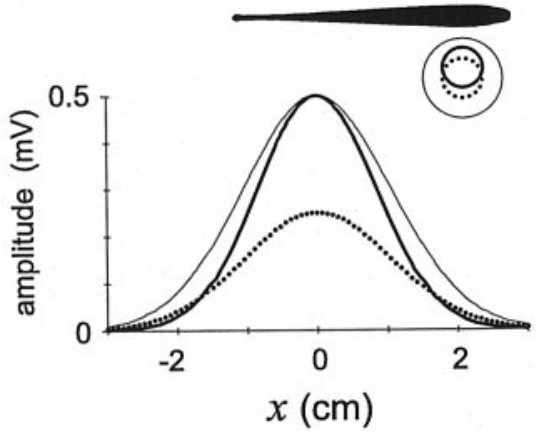

Figure 1. Computation of object distance in the electrosensory system. $a$, A schematic of the two-dimensional electric image on the surface of the fish for objects of two different sizes and lateral distances. Although the widths of the images are different, the peak amplitudes are the same (measured in grayscale, with white being the largest). Thus, detecting object distance based only on amplitude leads to ambiguities. $b$, One-dimensional slices of the electric images caused by conducting spheres of different sizes $\left(r_{o}=0.5 \mathrm{~cm}\right.$ and $\left.r_{o}=1.0 \mathrm{~cm}\right)$ and different lateral distances $\left(z^{*}=1.0 \mathrm{~cm}\right.$ and $z^{*}=$ $1.26 \mathrm{~cm}$ ). The schematic (top right, fish not to scale) illustrates the combinations of $r_{o}$ and $z^{*}$ (by line type, size, and location) that relate to the graph below. used to produce distinct behaviors (Metzner and Juranek, 1997). Here, we use theoretical analyses and modeling to investigate the influence of ELL pyramidal neuron tuning width in $2 \mathrm{D}$ on the accuracy of encoding object location in $3 \mathrm{D}$. In doing so, we suggest that the different ELL maps may be specialized for encoding the different stimulus features used for computing object distance.

\section{MATERIALS AND METHODS}

A model description of the electric image. The electric image caused by a spherical conductor is well approximated by a Gaussian-shaped surface with width and peak amplitude given by $2 \theta$ and $A_{o}$. Using data and simulations from a previous study (Rasnow, 1996), we have developed a parametric model of the electric image that enables our present analysis. We describe the electric image produced by a sphere of radius, $r_{o}$, at a location $\left(x^{*}, y^{*}, z^{*}\right)$ by the function $S$ (Eqs. 1-3):

$$
S(x, y)=A_{o} \exp \left(-\frac{\left(x-x^{*}\right)^{2}+\left(y-y^{*}\right)^{2}}{2 \theta^{2}}\right) .
$$

The half-width of the image $(\theta)$ increases linearly with lateral distance (Eq. 2) in the range of available data $\left(r_{o}=[0.125,0.7] ; z^{*}=[1.0,2.0] ; \theta\right.$, $r_{o}, x^{*}, y^{*}, z^{*}$ have units in centimeters). The peak amplitude of the image, $A_{o}$ (units in millivolts), decreases as the third power of lateral distance, and although it is actually proportional to the volume of the sphere (Rasnow, 1996), in the range considered, $A_{o}$ is approximately linear with $r_{o}$ (Eq. 3):

$$
\begin{aligned}
& \theta=c_{1}+c_{2} z^{*} \\
& A_{o}=\frac{r_{o}}{\left(z^{*}\right)^{3}} .
\end{aligned}
$$

With $c_{1}=(-0.055)$ and $c_{2}=(0.79)$, Equations 2 and 3 provide a good description of the data $\left(\chi^{2}<10^{-4}\right)$. This model is not meant to be a detailed reproduction of the electric image, but rather a simple description that allows us to gain insight into the nature of the electrosensory information available for electrolocation. The exact parameter choices do not affect our general conclusions.

A model of the ELL network. To describe the response of the population of ELL pyramidal neurons, we convolve the stimulus, $S$, with 2D Gaussian-shaped tuning curves of width, $\sigma$, and where $\left(x_{i} y_{j}\right)$ is the tuning curve (or receptive field) center of the neuron labeled $i j$. Because we have assumed that the electric image is also Gaussian, the convolution and hence the response of the pyramidal neuron population is given by Equation 4 (after rescaling to obtain a physiologically appropriate spike count for a $1 \mathrm{sec}$ time window, and accounting for a baseline activity level, $\left.g_{o}=100, E_{\text {baseline }}=20\right)$ (Bastian, 1986b). We include additive noise, $E_{\text {noise }}$, which has a normal distribution with zero mean and SD, $\eta$. The response of the neuron $i j, E_{i j}$, is described in Equation 5:

$$
\begin{gathered}
F_{i j}=E_{\text {baseline }}+g_{o} A_{o} \exp \left(-\frac{\left(x_{i}-x^{*}\right)^{2}+\left(y_{j}-y^{*}\right)^{2}}{2\left(\theta^{2}+\sigma^{2}\right)}\right) \\
E_{i j}=F_{i j}+E_{\text {noise }} .
\end{gathered}
$$

The network we consider consists of an $N \times N$ square grid of pyramidal neurons $(i, j=1, \ldots, N)$ with their locations on the grid defining the centers of their evenly spaced tuning curve centers $\left(x_{\dot{v}} y_{j}\right)$. Although we allow the grid size $N$ and the grid dimensions $(x, y)$ to vary, we specify the grid spacing $\left(x_{i+1}-x_{i}=y_{j+1}-y_{j}=\Delta=0.15 \mathrm{~cm}\right)$ so that the density of tuning curve centers $(\rho=46.7)$ is in the physiological range of $40-50$ neurons $/ \mathrm{cm}^{2}$ [expressed in relation to body surface area (Shumway, 1989a,b) (J. Lewis and L. Maler, unpublished observations)]. The center of the grid is the origin, $(x, y)=(0,0)$. Although receptive field sizes of ELL pyramidal neurons have been reported previously (Bastian, 1981; Shumway, 1989a), the methods used (different combinations of object size and direct electrical stimulation) make it difficult to directly obtain values of $\sigma$. However, estimates for the physiological range of $\sigma$ are between $\sim 0.3$ and $0.7 \mathrm{~cm}$ depending on the particular ELL map (the centromedial map has the narrowest, and the lateral map has the widest tuning curves).

For simulations of this network, we calculate a neuron response profile using Equation 4 for a given set of object features. Gaussian random numbers $\left(E_{\text {noise }}\right)$ with zero mean and SD of $\eta$ are generated (Press et al., $1993)$ for each neuron and added to the response profile $E_{i j}$. These responses are then rounded to the nearest integer value to give the single trial response of the population in terms of spike count. A typical single trial response is shown in Figure $2 c$. For the open symbols plotted in Figure 3, we estimate the image features from this noisy profile using a least-squares fit to Equation 4 with the free parameters being either $r_{o}, x^{*}$, $y^{*}, z^{*}$ (Fig. $3 a$ ) or $\theta, A_{o}, x^{*}, y^{*}$ (Fig. $3 b$ ). This is equivalent to a maximum-likelihood (ML) estimate of the free parameters (Kay, 1993; Deneve et al., 1999). The estimation error over a number of trials is given by the mean-squared difference between the estimated and true values of each parameter (equivalent, in this case, to the variance of the estimated values). For all of the results shown, we use additive noise (Eq. 5) with $\eta=7$ in agreement with preliminary data (J. Bastian, J. Lewis, and L. Maler, unpublished data); however, the exact value of $\eta$ does not affect our conclusions.

We consider two different network implementations of a two-step algorithm for determining $\theta$ and $A_{o}$; one in which the same network is used to estimate both stimulus features (model 1) and the other consisting of two networks (model 2), with each used to estimate a single feature (see Results) (see Fig. 5). Our initial comparison involves specific, previously proposed (Assad et al., 1999), mechanisms to implement the algorithm, but to also compare these models in a general decoding framework we used a variation of the ML method described earlier (see Results). In this case, two networks of the same size $(41 \times 41$ grid $)$ were used. The first network was used to estimate $A_{o}$ by using a least-squares fit to the noisy neuronal profile with $A_{o}$ and $\theta$ as free parameters. A similar procedure was then performed on the second network but with only $\theta$ as a free parameter, with $A_{o}$ fixed to the value estimated by the first network. This can be viewed as an optimal implementation of the two-step algorithm.

The Cramer-Rao lower bound and Fisher information. Estimation of object size and location in the present context is formulated as the estimation of a vector parameter, $\varphi=\left(\varphi_{1}, \varphi_{2}, \varphi_{3}, \varphi_{4}\right)$. In Figure $3 a$, $\left(\varphi_{1}, \varphi_{2}, \varphi_{3}, \varphi_{4}\right)$ corresponds to $\left(r_{o}, x^{*}, y^{*}, z^{*}\right)$, whereas in Figure $3 b$ $\left(\varphi_{1}, \varphi_{2}, \varphi_{3}, \varphi_{4}\right)$ equals $\left(\theta, A_{o}, x^{*}, y^{*}\right)$. The accuracy of an estimator can be assessed by its bias and variance. An estimator is considered unbiased if its average value is equal to the true value of the estimated parameter. 


\section{a}
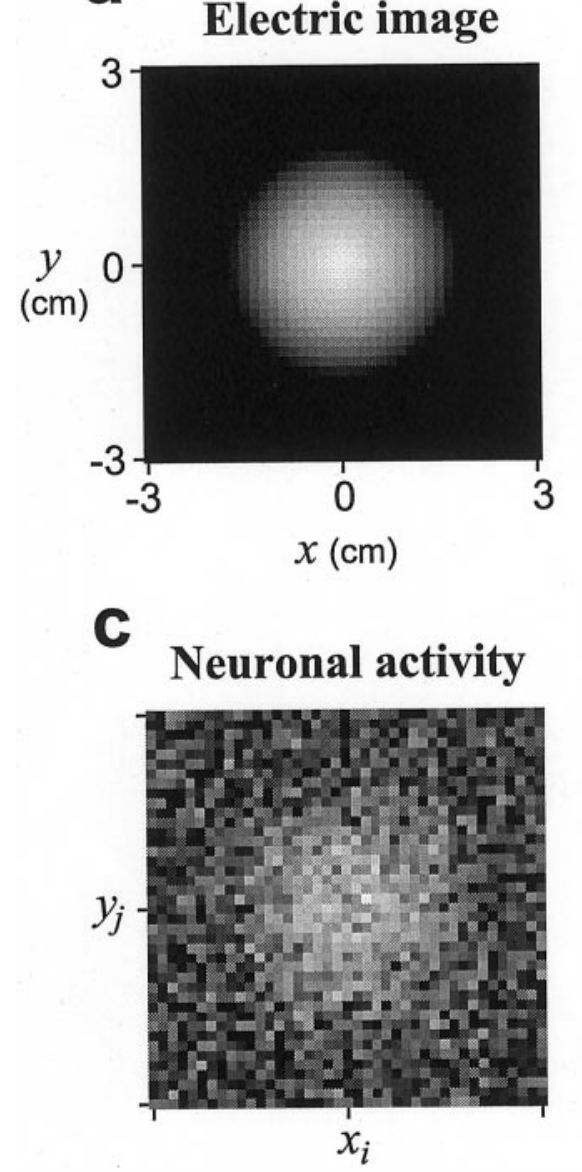

b

\section{Neuronal grid}

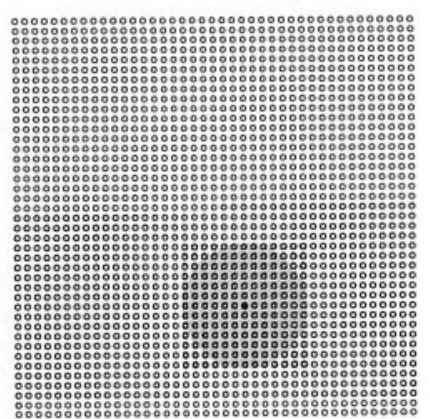

d

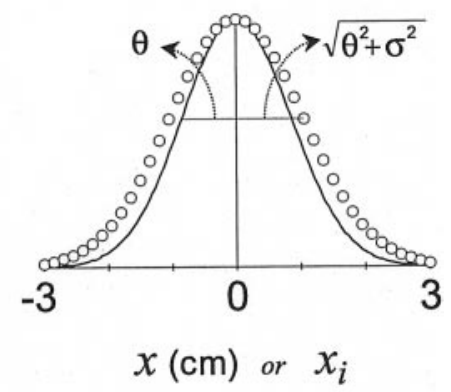

Figure 2. A model of the ELL network response. $a$, The electric image for an object of radius $\left(r_{o}=0.5 \mathrm{~cm}\right)$ at a location $\left(x^{*}, y^{*}, z^{*}\right)=(0,0,1)$ is shown on a spatial grid. Image amplitude is in grayscale (white $=0.3 \mathrm{mV}$; black $=0$ $\mathrm{mV})$. $b$, The $41 \times 41$ neuronal grid with the tuning curve size of one neuron denoted by the gray shaded circle ( $\sigma=$ 0.6). The position of each neuron on the grid is given by its tuning curve center $\left(x_{i}, y_{j}\right)$ in register with the image in $a$. The neuronal density is $\rho=46.7$ neurons $/ \mathrm{cm}^{2} . c$, A typical realization of the neural response produced by the image in $a$ is shown in grayscale (white $=65 \mathrm{~Hz}$; black $=0 \mathrm{~Hz}$ ). Other parameter values are: $E_{\text {baseline }}=20 ; g_{o}=100 ; \eta=7 . d$, The broadening of the average neuronal response (plotted vs the tuning curve centers $x_{i}$, open circles) compared with the electric image (solid line) illustrated in one-dimension for the above parameters. The half-widths of the image and response profile are $\theta$ and $\sqrt{ }\left(\theta^{2}+\sigma^{2}\right)$, respectively.
The variance of an unbiased estimator is equivalent to the mean-squared estimation error; the lower the variance the more accurate the estimator. The theoretical lower limit on the variance of any unbiased estimator is given by the Cramer-Rao lower bound (Kay, 1993). The Cramer-Rao bound is the reciprocal of the Fisher information, $I_{F}$ (Eqs. 6, 7). The more accurate an estimator is, the more information it provides about the parameter that is estimated; this information is quantified by $I_{F}$ :

$$
\begin{gathered}
\operatorname{var}\left(\varphi_{k}^{e s t}\right) \geq\left[I_{F}^{-1}(\varphi)\right]_{k k} \\
{\left[I_{F}(\varphi)\right]_{k m}=\frac{1}{\eta^{2}} \sum_{i=1}^{N} \sum_{j=1}^{N} \frac{\partial F_{i j}}{\partial \varphi_{k}} \frac{\partial F_{i j}}{\partial \varphi_{m}}}
\end{gathered}
$$

In Equations 6 and 7, $\varphi^{\text {est }}$ is the estimate and $\varphi$ is the true value of the vector parameter, $\eta$ is the $\mathrm{SD}$ of $E_{\text {noise }}, N^{2}$ is the number of neurons in the population, $k=(1, \ldots, 4)$, and $m=(1, \ldots, 4)$ for each of the four parameters. Thus, when four parameters are estimated simultaneously, $I_{F}$ is a $4 \times 4$ matrix. The Fisher information has previously been used to measure the accuracy of neuronal population codes (Abbott and Dayan, 1999; Deneve et al., 1999; Zhang and Sejnowski, 1999). Assuming ( $x^{*}=$ $0, y^{*}=0$ ) the Fisher information for the parameter $\theta$ alone can be rewritten in terms of the grid spacing, $\Delta$ (Eq. 8):

$$
I_{F}(\theta)=\frac{1}{\eta^{2}} \sum_{i=1}^{N} \sum_{j=1}^{N}\left(\frac{\partial}{\partial \theta} g_{o} A_{o} \exp \left(-\frac{(i \Delta)^{2}+(j \Delta)^{2}}{2 \theta^{2}+2 \sigma^{2}}\right)\right)^{2} .
$$

In situations in which multiple but similar neuronal populations are involved in estimation (e.g., multiple maps), the Cramer-Rao bound can be calculated from the Cramer-Rao bound for the individual networks. If $\theta_{1}$ and $\theta_{2}$ are estimates from the two different networks, and the com- bined estimate is $\theta_{1-2}$, then the variance of $\theta_{1-2}$ can be described by Equation 9 (Rosner, 1995):

$$
\begin{gathered}
\theta_{1-2}=\frac{k_{1} \theta_{1}+k_{2} \theta_{2}}{k_{1}+k_{2}} \\
\operatorname{var}\left(\theta_{1-2}\right)=k_{1}^{2} \operatorname{var}\left(\theta_{1}\right)+k_{2}^{2} \operatorname{var}\left(\theta_{2}\right) .
\end{gathered}
$$

In the case of two identical networks (i.e., same size and same tuning widths, etc), taking the average of the two independent estimates is optimal; in this case $k_{1}=k_{2}=0.5$, and because $\operatorname{var}\left(\theta_{1}\right)=\operatorname{var}\left(\theta_{2}\right)$, the net Cramer-Rao bound is exactly half that for the individual networks. To similarly evaluate the combinations of networks with different properties (as in Fig. 7), a weighted average is best, so we choose the constants $k_{1}$ and $k_{2}$ to be the reciprocals of the single network variances $\left[k_{1}=\right.$ $1 / \operatorname{var}\left(\theta_{1}\right)$ and $\left.k_{2}=1 / \operatorname{var}\left(\theta_{2}\right)\right]$. A similar procedure was used for $A_{o}$ estimates as well.

\section{RESULTS}

\section{Estimating object distance}

From a 2D electric image on their body surface, electric fish are able to determine the 3D location $\left(x^{*}, y^{*}, z^{*}\right)$ of the object producing the image (von der Emde et al., 1998). The object location in the body plane ( $x-y$ plane) can be estimated from the location at which the image has its peak amplitude. However, the peak amplitude of the electric image provides ambiguous information about the third dimension, lateral distance away from the fish $\left(z^{*}\right)$. In Figure $1 a$, two spherical objects of different sizes (and otherwise identical) are located at the same $\left(x^{*}, y^{*}\right)$ location, but the larger object is farther away. For this, and many other com- 

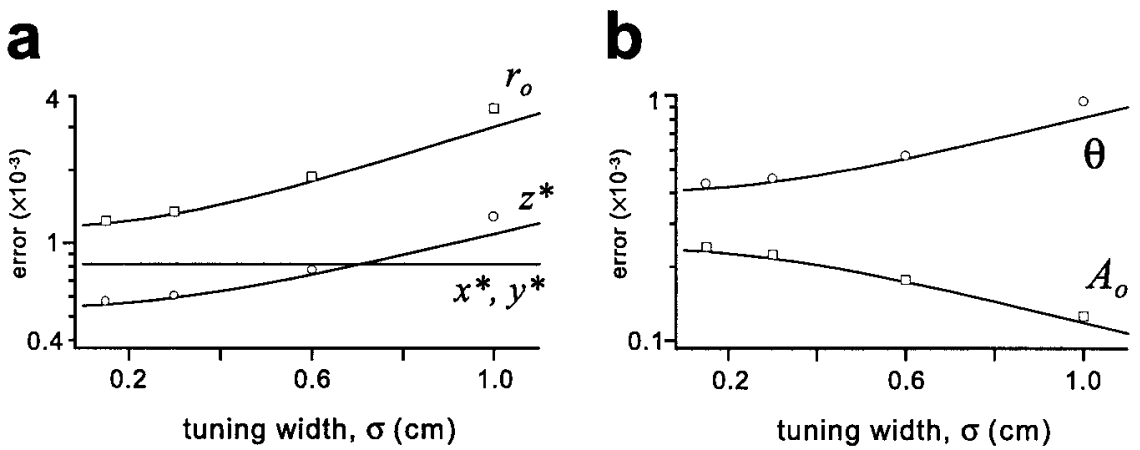

Figure 3. Accuracy of estimating electric image features. $a$, The error in estimating the size $\left(r_{o}\right)$ and $\left(x^{*}\right.$, $\left.y^{*}, z^{*}\right)$ location of a conducting sphere as a function of pyramidal neuron tuning width, $\sigma . b$, The error in estimating the corresponding image features $A_{o}$ and $\theta$ as a function of tuning width, $\sigma$. In both panels, the continuous lines indicate the analytically computed error given by the minimum variance of the estimate (Cramer-Rao lower bound, see Results). The open squares and open circles [for $r_{o}(a)$ and $z^{*}(b) A_{o}$ and $\theta$, respectively] show the errors from network simulations (5000 trials each point; $41 \times 41$ neuronal grid; $\rho=46.7$ neurons $/ \mathrm{cm}^{2} ; E_{\text {baseline }}=20 ; g_{o}=100$; $\eta=7$; see Results). For the theoretical calculations, a larger grid $(101 \times 101)$ was used (with the neuronal density preserved) to avoid edge effects for the larger tuning widths. For the features $r_{o}, A_{\mathrm{o}}$, and $z^{*}$, the error is normalized to the true values of the feature. The true values are $r_{o}=0.5, A_{o}=0.289, \theta=1.00$, and $\left(x^{*}, y^{*}, z^{*}\right)=(0,0,1.2)$.

binations of object size and lateral distance, the peak amplitude of the image is the same and thus cannot be used to unambiguously determine the lateral distance of each object (see Materials and Methods; Eq. 3) (Rasnow, 1996). The image produced by the larger object is wider than the other (Fig. 1a). This is shown more clearly by a one-dimensional slice through the image (Fig. 1b). When the image is normalized to its peak amplitude, its width can then be used to estimate lateral distance, $z^{*}$ (Rasnow, 1996; Assad et al., 1999).

To enable our analyses, we used a simplified description of the electric image. We assume the electric image has a 2D Gaussian shape, with its peak amplitude and half-width given by the parameters $A_{o}$ and $\theta$ (see Materials and Methods) (Eqs. 1-3). Because $\theta$ provides a measure of normalized width and varies linearly with lateral distance, $z^{*}$ (Rasnow, 1996), it then can be used to estimate lateral distance (we use $\theta$ and image width interchangeably, although $\theta$ is actually the half-width).

Another image feature proposed as an indicator of object distance is the maximum slope of the image normalized to its peak amplitude (von der Emde et al., 1998; von der Emde, 1999). For a Gaussian image, this quantity varies as $1 / \theta$ and also fits the published maximum slope data (von der Emde et al., 1998) very well (Lewis and Maler, unpublished observations). Because of the direct relationship between $\theta$, maximum slope, and previously reported data, we have discussed our results in terms of $\theta$ alone.

\section{Estimation accuracy and tuning curve width}

In the present context, downstream electrosensory networks must extract information about object location given a noisy profile of activity in the ELL pyramidal neuron population. We have formulated a simple model of the ELL population response to a stereotyped electric image (see Materials and Methods). Figure $2 a$ shows the electric image produced by a small sphere (Eq. 1), which provides the input to the $2 \mathrm{D}$ grid of model neurons that constitute the ELL network (Fig. 2b). Each neuron on the grid integrates input from the electric image over a restricted range or receptive field (shown schematically by the shaded region in Fig. $2 b$; Eq. 4), such that for a point stimulus each neuron has a $2 \mathrm{D}$ Gaussian-shaped tuning curve (in the $x-y$ plane). The tuning width is given by $2 \sigma$ (measured at a height corresponding to $e^{-1 / 2}$ of the tuning curve peak). We ignore any contributions that dynamics may provide, with the response of each neuron given by a spike count over an integration time of $1 \mathrm{sec}$. After the addition of noise the ELL population response profile resembles a noisy replication of the electric image (Fig. 2c). Because the electric image is not a point stimulus, the actual response profile of the ELL population is wider than the image, to an extent that depends on the relative values of $\theta$ and $\sigma$ (Fig. 2d) (see next section).

Given the noisy response profile of the ELL population, the typical population decoding problem is to determine the features of the object $\left(x^{*}, y^{*}, z^{*}, r_{o}\right)$ that produced the response (Abbott, 1994; Salinas and Abbott, 1994; Deneve et al., 1999; Zhang and Sejnowski, 1999). As discussed before in a functional context, to unambiguously determine $z^{*}$ and $r_{o}$, two features of the electric image produced by the object must be estimated: the image width and the amplitude of the image peak ( $\theta$ and $A_{o}$, respectively). The accuracy of estimation is limited by the accuracy with which the ELL neurons jointly encode these different features. Using a common approach from statistical estimation theory (Kay, 1993), we can determine an upper limit on this accuracy by computing the Cramer-Rao lower bound for estimating each object feature $x^{*}, y^{*}, z^{*}$, and $r_{o}$, as well as the image features $\theta$ and $A_{o}$ (Eq. 6) (see Materials and Methods). Accuracy in this context is given by the mean-squared estimation error, or equivalently, the variance of the estimate. We investigated the influence of two parameters on estimation accuracy: the lateral distance of the object $z^{*}$ and the tuning curve width $\sigma$. The error bound for estimating all features increases with $z^{*}$ (data not shown). This is not surprising because the image amplitude (and thus the effective signal-tonoise ratio) decreases fairly quickly with distance (Eq. 3). More interestingly, the effects of changing $\sigma$ differ between the features (Fig. 3). There is no effect on estimating the $x^{*}-y *$ location (Fig. $3 a$ ); the same result has been found previously for point stimuli (Snippe and Koenderink, 1992; Abbott and Dayan, 1999; Zhang and Sejnowski, 1999). On the other hand, increasing tuning width $\sigma$ results in worse estimation of $r_{o}$ and $z^{*}$ (Fig. 3a). For estimating electric image features (Fig. $3 b$ ), increasing $\sigma$ results in worse estimation of $\theta$ (larger error), but better estimation of $A_{o}$ (smaller error). Intuitively, this makes sense, wider tuning curves allow more neurons to accurately contribute to the estimation of $A_{o}$, and by averaging across neurons, a better estimate results. Estimating image width is different although, because the ELL neurons distort the image through a convolution with their tuning curves (Eq. 4, Fig. 2d). This distortion increases with tuning width, resulting in more neurons that do not accurately represent image width, nonetheless influencing the $\theta$ estimate.

Shown also in Figure 3 are the results of network simulations. Using a network grid consisting of a physiological number and density of neurons $(41 \times 41$ neuronal grid, density $\rho=46.7$ neurons $/ \mathrm{cm}^{2}$ ), we estimated the image features from the noisy neural responses using an ML approach (see Materials and Methods). The estimation error for this method is very close to the 
a

\section{Estimating $A_{o}$}

Figure 4. Two-step algorithm for estimating electric image width. $a$ and $b$ show representative response profiles $E_{i}$ of a one-dimensional slice through the neuronal grid. In $a$ the peak amplitude of the image is estimated by the average activity $E_{\text {ave }}$ of all neurons firing above a threshold level of $E_{\text {baseline }}+\phi_{\mathrm{a}}$ $\left(E_{\text {baseline }}=20\right)$. In $b$ the response profile is normalized by $E_{\text {ave }}$, and the image width is estimated by the number of neurons $N_{\mathrm{w}}$ firing above a threshold level of $\left(E_{\text {baseline }} / E_{\text {ave }}\right)+\phi_{\mathrm{w}} \cdot c$, The average spike count of the neurons above threshold $\left(E_{\text {ave }}\right)$, as well as the decay in actual peak activity $\left(g_{o} A_{o} ; g_{o}=100\right)$ with increasing object distance $z^{*}\left(\phi_{\mathrm{a}}=2 \eta=14 ; \sigma=\right.$ $0.6)$. Over this range of $z^{*}, E_{\text {ave }}$ varies linearly with $A_{o}$ (inset). $d$, The fraction of total neurons activated above a threshold level of $\phi_{\mathrm{w}}=\mathrm{e}^{-1 / 2}$ (i.e., the number of neurons with a preferred location within a radius $\theta$ of the object location) plotted versus $\theta$. For different values of $\sigma$, this measure increases in an almost linear manner with $\theta$. The solid lines are the theoretical curves derived for a continuous distribution of neurons (see Results), and the open symbols show the measure for an actual model network $(41 \times$ 41 neuronal grid; $\rho=46.7$ ).

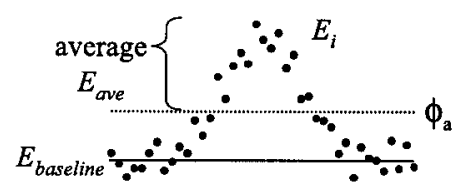

b Estimating $\theta$
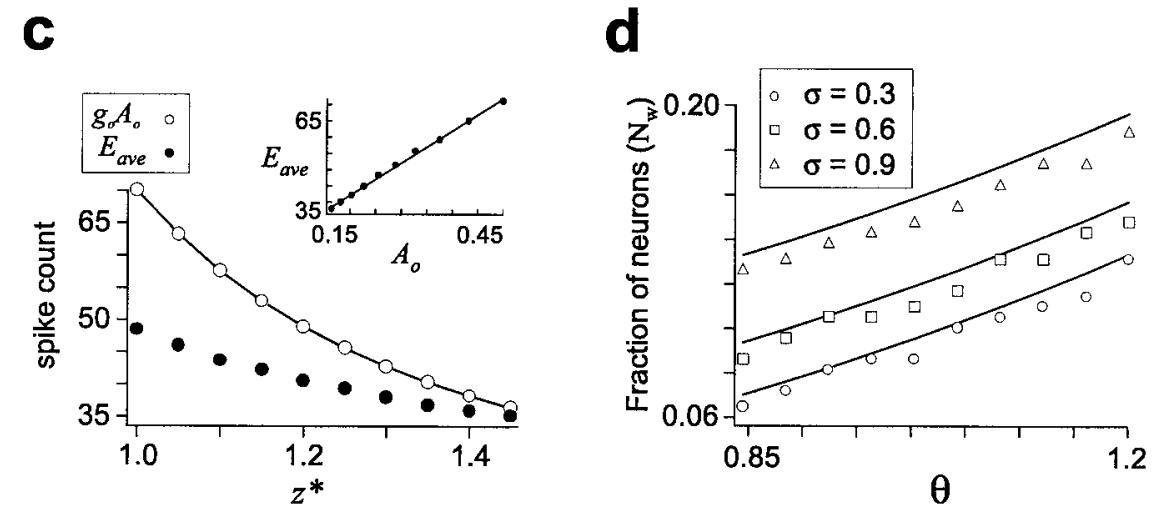

corresponding lower bound (Fig. 3, compare open symbols with solid lines). Note that for larger $\sigma$ however, there is a slight deviation from the theoretical bound attributable mainly to edge effects (i.e., the neuronal image has above baseline values beyond the limits of the grid edges).

The relationship between the accuracy of image width estimation and tuning width can be made explicit by expressing the Fisher information for $\theta, I_{F}(\theta)$, in terms of the grid spacing, $\Delta$, the distance between tuning curve centers (Eq. 8). Differentiating $I_{F}(\theta)$ with respect to $\sigma$ reveals that $I_{F}(\theta)$ decreases with $\sigma$ (i.e., the derivative is negative and thus the estimation error increases) as long as $\theta^{2}+\sigma^{2}>(\Delta / 2)^{2}$. This condition will hold as long as the tuning curve width is greater than the grid spacing (i.e., if $2 \sigma>$ $\Delta)$. A similar calculation shows that the Fisher information for $A_{o}$, $I_{F}\left(A_{o}\right)$, increases with $\sigma$ for all $\sigma>0$.

\section{A simple neural algorithm for determining object distance}

Estimating $\theta$, in the present context, is equivalent to estimating the half-width of the image at a level of $\mathrm{A}_{o} e^{-1 / 2}$. To provide an unambiguous estimate of $z^{*}$, a measure of image width must be calculated from an image normalized by $A_{o}$. So in such a practical situation, peak amplitude $A_{o}$ must be estimated first, before image width.

One simple algorithm to calculate image width is to first normalize the neural responses to the maximal response and then count the number of neurons that are active above a certain threshold (Assad et al., 1999). One way to formalize this two-step algorithm is to first compute the average activity $E_{\text {ave }}$ of all the neurons firing above a threshold, $\phi_{\mathrm{a}}$ (Fig. 4a). This step (step 1) provides an estimate of the peak response in the population, which can be used to normalize all neural activity. Then in step 2, the fraction of neurons $\left(N_{\mathrm{w}}\right)$ firing above a different threshold $\left(\phi_{\mathrm{w}}\right)$ can be determined (Fig. $\left.4 b\right)$. These two thresholds are distinct in that $\phi_{\mathrm{a}}$ is fixed and not relative to any neural response, whereas $\phi_{\mathrm{w}}$ comes after the normalization step and is relative to the maximum response in the network. Figure $4, c$ and $d$ shows how these measures vary with the features they are supposed to estimate. The actual peak neural activity $\left(g_{o} A_{o}\right)$ differs from $A_{o}$ by a constant factor and thus varies with $z^{*}$ in parallel with $A_{o}$ (Fig. 4c). However, $E_{\text {ave }}$ underestimates $g_{o} A_{o}$ but still varies linearly with $A_{o}$ (Fig. $4 c$, inset). Similarly, $N_{\mathrm{w}}$ varies in a near linear manner with $\theta$ (Fig. $4 d$ ).

We consider two specific neural implementations of this algorithm (Fig. 5). Model 1 uses the same map (i.e., network), with tuning width $\sigma_{1}$, for estimating both $\theta$ and $A_{o}$. Model 2 uses two maps, one with a relatively large tuning width $\left(\sigma_{2}=1\right)$ for estimating $A_{o}$ and another with narrower tuning widths $\left(\sigma_{1} \leq 1\right)$ for estimating $\theta$. Model 1 is analogous to a single sensory map for all computations, and Model 2 is analogous to having two specialized sensory maps, one for estimating peak amplitude $A_{o}$, with larger tuning widths, and the other for estimating width $\theta$, with smaller tuning widths. It is critical to note that both models use the same number of neurons in each processing step (each map is a $41 \times 41$ neuronal grid). The critical difference is that model 2 has two different tuning widths for each processing step.

To compare the performance of these models, we compute $N_{\mathrm{w}}$ for many simulated presentations of an object over a range of values of $\sigma_{1}$ and $z^{*}\left(\phi_{\mathrm{a}}=2 \eta ; \phi_{\mathrm{w}}=\mathrm{e}^{-1 / 2}\right)$. In this situation, the true value of $N_{\mathrm{w}}$ is given by the number of neurons with preferred locations within a circle of radius $\theta\left(\pi \theta^{2} \rho\right)$. The estimate of $N_{\mathrm{w}}$ from the present neural algorithm, however, is biased. This is in part because of its dependence on $E_{\text {ave }}$ and also because it is determined from a neural profile that has an effective width of $\sqrt{ }\left(\theta^{2}+\sigma^{2}\right)$ caused by the tuning curve convolution (Eq. 4, Fig. $2 d$ ). Because in the context of these models, downstream networks would have to use $N_{\mathrm{w}}$ to estimate object distance, and $N_{\mathrm{w}}$ is directly related to $\theta$ and object distance (Fig. $4 d$ ), we evaluate model performance from the bias and variance in $N_{\mathrm{w}}$. In all cases tested $\left(\sigma_{1}=0.15-1.0 ; z^{*}=1.0-1.4\right)$, model 2 outperforms model 1. For $\mathrm{z}^{*}=1.2$, the estimation variance for both models is shown in Figure $6 a$. The biases in $N_{\mathrm{w}}$ estimation are nearly identical for both models (data not shown), but the variance for model 2 is substantially less than that for model 1 . Because model 2 is better at estimating peak amplitude (by virtue of its wide tuning curves 


\section{Model 1}

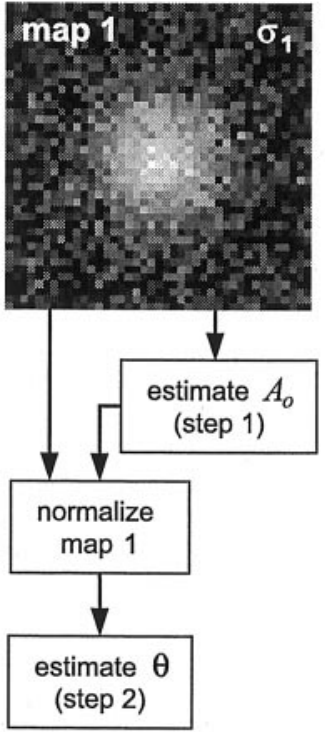

Model 2

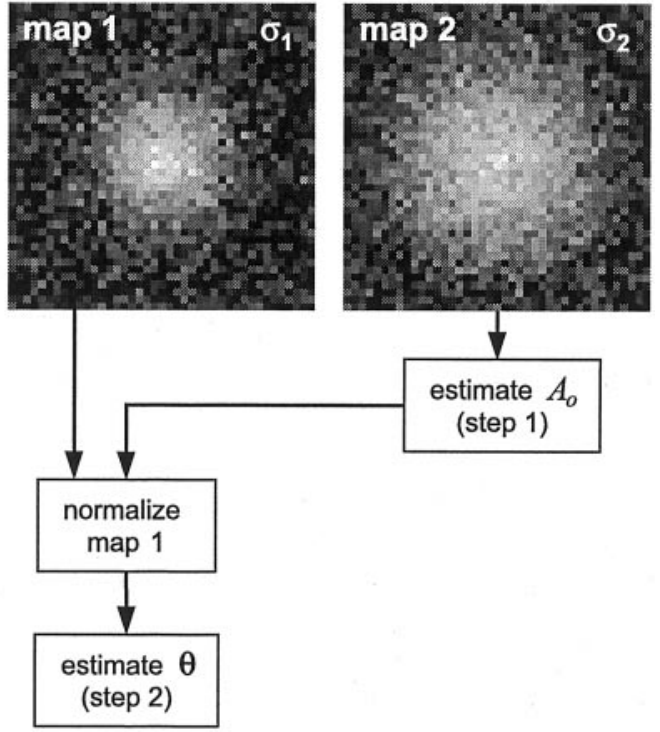

Figure 5. Schematic representation of two models for implementing the two-step algorithm for estimating electric image width. In model 1 (left), both $A_{o}$ and $\theta$ are estimated using the same map, map 1 with tuning widths $\sigma_{1}$. In model 2 (right), separate maps are used for each estimation step: map 2, with wide tuning curves $\left(\sigma_{2}=\right.$ 1 ), is used to obtain an estimate of $A_{o}$, which is then used to normalize the activity in map 1 , with narrow tuning curves $\left(\sigma_{1}<\sigma_{2}\right)$, from which an estimate of $\theta$ is obtained. Because $A_{o}$ and $\theta$ are estimated separately in this two-step algorithm, both models use the same number of neurons to estimate each feature, although model 2 has two maps, and model 1 has only one. for this step, $\sigma_{2}=1$ ), the variance is dominated by the width estimator, and thus increases with $\sigma_{1}$ in the same manner as the Cramer-Rao bound for $\theta$ (Fig. 3b). Model 1 must use a network with the same $\sigma_{1}$ for all steps, so there is a trade-off between accuracy of peak amplitude estimation and accuracy of width estimation. Peak amplitude estimation is better for larger $\sigma_{1}$ when more neurons are activated close to peak levels, but width estimation is better for smaller $\sigma_{1}$. In the case shown (Fig. $6 a$ ), the amplitude estimate dominates even for small $\sigma_{1}$ and thus the overall variance decreases with $\sigma_{1}$, similar to the Cramer-Rao bound for $A_{o}$ (Fig. $3 b$ ). When $\sigma_{1}=1$, both models have similar overall accuracy. Although it would seem that model 2 effectively has twice as many neurons as model 1 , as stated earlier, it really uses the same number of neurons as model 1 for each processing step. The slightly better performance of model 2 for $\sigma_{1}=1$ is caused by the independence of the responses between the different maps. In other words, if the noise in the neuronal responses was exactly correlated between the two maps of model 2, the accuracy would be identical to that of model 1 .

The general trends shown in Figure $6 a$ are similar for neuronal densities within $\sim 50-150 \%$ of that used in the simulations shown. We also tested several combinations of values for $\phi_{\mathrm{a}}$ (range, $2 \eta-4 \eta$ ) and $\phi_{\mathrm{w}}$ (range, $\left.0.25-0.75\right)$ for $z^{*}=1.2$ and $\sigma_{1}=0.3$.
Similar trends resulted, so the increased accuracy of model 2 over model 1 does not depend critically on these threshold values. In addition, we also considered conditions in which the noise term $E_{\text {noise }}$ was such that the SD of the ELL neuron responses was equal to their mean response $F_{i j}$ (Eq. 4), rather than constant $(\eta=7)$ and independent of $F_{i j}$. This type of noise resulted in similar results (data not shown) and does not change our conclusions.

Although the previous analysis demonstrates a clear difference between the two models, it is important to prove that this difference is fundamental and is not simply attributable to the details of the algorithm implementation or the fact that model 2 uses two independent networks. We now consider two independent maps composed of $41 \times 41$ neuronal grids with tuning widths of $\sigma_{1}$ and $\sigma_{2}$, respectively. Map 2 provides an estimate of $A_{o}$ using ML estimation; this estimate is used to normalize the activity in map 1. Then map 1 is used to find the ML estimate of $\theta$. For model 1 , both maps have the same tuning width $\sigma_{1}=\sigma_{2}$. Model 2 is identical to model 1 except that map 2 has a fixed tuning width $\sigma_{2}=1$. This constitutes a test of the two models in a general decoding framework in which the only difference is in the tuning width of map 2. The results are similar to those previous, with model 2 providing a better estimate of $\theta$ (Fig. $6 b$ ). In this case a

\section{Neural implementation} (two-step algorithm)

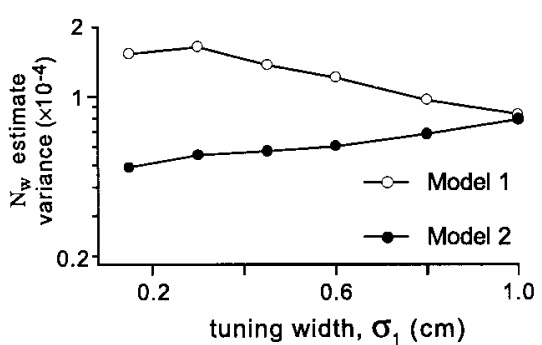

$\mathbf{0}$

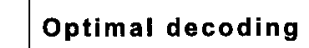
(two-step algorithm)

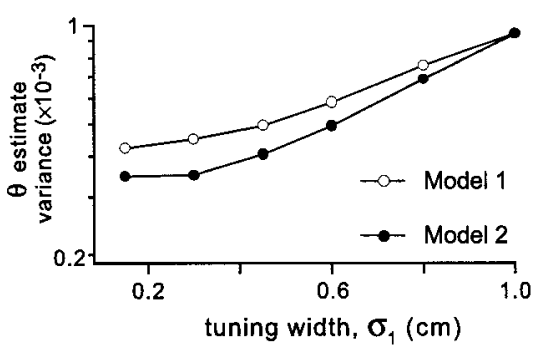

Figure 6. Performance of the two models in implementing the two-step algorithm for estimating electric image width. $a$, This panel shows the variance in the estimate of $N_{\mathrm{w}}$ for each model. Model 1 (open symbols) results in higher variance than model $2\left(\sigma_{2}=1\right.$; closed symbols) for all tuning widths $\sigma_{1} . N_{\mathrm{w}}$ is the fraction of neurons above threshold (i.e., the actual number normalized by the total number of neurons $N^{2}$; parameter values are $N=41$, $\phi_{\mathrm{w}}=\mathrm{e}^{-1 / 2}, \phi_{\mathrm{a}}=2 \eta, r_{o}=0.5,\left(x^{*}, y^{*}, z^{*}\right)=(0$, $0,1.2), \rho=46.7, E_{\text {baseline }}=20, g_{o}=100, \eta=7$. The true value of $N_{\mathrm{w}}=\pi \theta^{2} \rho / N^{2} \sim 0.09$. $b$, The variance of the $\theta$ estimate for a generalized decoding scheme in both models (see Results). All parameter values are the same in $a$ and $b$, except that in $b$ two independent maps are used for both models (see Results). Note that when $\sigma_{1}=1$ in this case, both models are identical so the variances are necessarily the same. Each point in $a$ and $b$ represents the variance calculated from 3000 simulated trials. 
a

Figure 7. Performance of two maps in the context of the Cramer-Rao bound. For two different combinations of two maps (similar to those in Fig. $6 b$ ), the analytically calculated Cramer-Rao bounds for estimating $A_{o}(a)$, and $\theta(b)$ (see Materials and Methods), are shown by the thick solid and dotted lines, respectively. In one configuration $\left(\sigma_{2}=\sigma_{1}\right)$, both maps have the same tuning width, and in the other configuration $\left(\sigma_{2}=1\right)$, one map has a tuning width of $\sigma_{1}$, and the other is fixed at $\sigma_{2}=1$. Also shown (thin solid lines) are the analytically calculated Cramer-Rao bounds for a single map (same as those in Fig. $3 b$ ).

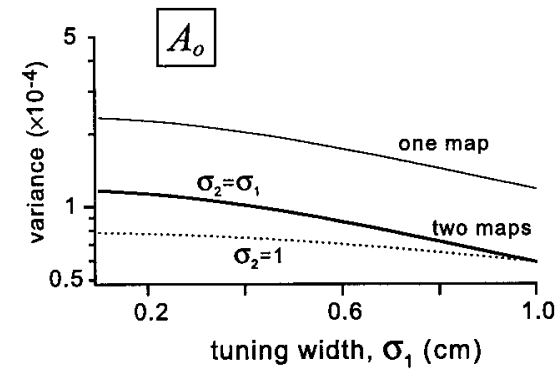

b

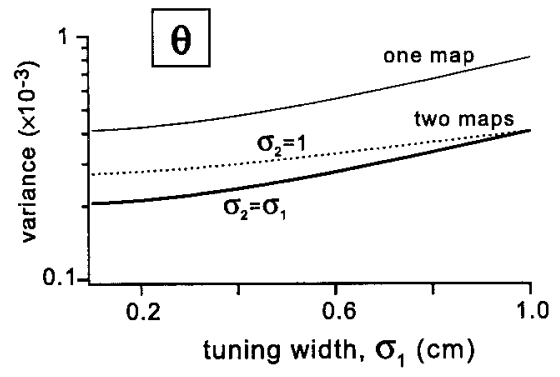

however, the error increases with $\sigma_{1}$ for both model 1 and 2, in the same manner as the Cramer-Rao bound for $\theta$ (Fig. $3 b$ ), suggesting that $\theta$ estimation dominates the overall estimation error. This provides a theoretical validation of our conclusions, but it is certainly not an option for the fish. The ELL does not have multiple maps with the same tuning widths, and thus the fish does not have access to identical information from two identical maps. Our initial analysis (Fig. $6 a$ ) shows how the specialized use of an additional map can improve the computation performed by a single map.

The two-step algorithm we have considered is based on previous ideas (Assad et al., 1999) and practical constraints (i.e., peak amplitude must be estimated before normalization can occur). But it is also interesting to ask how maps can be combined in the context of optimal estimation as defined by the Cramer-Rao bound. We again consider the two-map configuration analyzed in Figure $6 b$. We calculated the CramerRao bounds for $\theta$ and $A_{o}$ for two maps (see Materials and Methods) and compared them to that for a single map (Fig. $7 a, b)$. Two maps with identical tuning widths are twice as good as one map with that tuning width (i.e., the error decreases by half for two maps). The neuronal density is a critical factor in determining population coding accuracy (Zhang and Sejnowski, 1999). Having two identical maps is the same, in terms of accuracy, as having a single map with twice the density, not necessarily twice the number of neurons. Also shown in Figure 7 is that having one of the maps with a fixed tuning width $\left(\sigma_{2}=1\right)$ is better than two identical maps for estimating $A_{o}$, but worse for estimating $\theta$. Thus, the relative importance of these parameters will influence the optimal configuration of the two maps; if a premium is placed on estimating $\theta$ independently of $A_{o}$, then narrow tuning in both maps is better. In the two-step algorithm considered in this paper, accurately estimating $A_{o}$ is critical for the overall accuracy of estimating $\theta$, so a combination of tuning widths is best.

\section{DISCUSSION}

\section{Multiple maps and population coding}

Weakly electric fish can accurately electrolocate objects in their surroundings using sensory information contained in a $2 \mathrm{D}$ electric image (Bastian, 1987; Heiligenberg, 1991; Nelson and MacIver, 1999; von der Emde, 1999). To unambiguously extract 3D object location, the fish must compute the width and location of the peak of the electric image that is normalized to its peak amplitude (Rasnow, 1996). The electric image is initially encoded in four populations of pyramidal neurons that comprise the four parallel maps in ELL. One map (ampullary system) is specialized for low-frequency signals. The neurons within the three remain- ing maps (tuberous system) can be distinguished, among other characteristics, by their distinct spatial response properties: the lateral map with large receptive fields, centromedial map with small receptive fields, and the centrolateral map with intermediate-sized receptive fields (Shumway, 1989a,b; Metzner, 1999; Turner and Maler, 1999). Our results suggest a novel function for the parallel sensory maps in ELL, as well as the occurrence of parallel maps in other sensory systems. Namely, in addition to coarse coding stimulus features on different scales, parallel sensory maps may also be optimized to encode features of a sensory stimulus to which the component neurons are not tuned in the same manner. Specifically, in addition to encoding the $2 \mathrm{D}$ electric image at different spatial scales, different ELL maps can also be specialized to accurately represent the sensory features required to compute the third dimension, i.e., object distance.

Previous theoretical studies have found that neuronal tuning width (or spatial resolution) should not affect encoding accuracy in 2D (Snippe and Koenderink, 1992; Abbott and Dayan, 1999; Zhang and Sejnowski, 1999). This is also the case for the coarsecoded features of a spatially extended stimulus (i.e., the electric image) (Fig. 3a). This result does not apply when multiple 2D stimuli are given simultaneously, as in two-point discrimination, where narrower tuning curves are better (Snippe and Koenderink, 1992). We show that depending on the encoding strategy for a particular stimulus feature, either wider or narrower tuning curves improve encoding accuracy. We illustrate the impact of tuning width on the accuracy of determining object distance from the electric image using two simple models. To encode the peak amplitude of the electric image, wider tuning curves in the two coarse-coded dimensions ( $x$ and $y$ ) result in higher accuracy; whereas, to encode image width, narrower tuning curves are more accurate. This suggests that the lateral map in ELL (in addition to its other functions, such as processing high-frequency signals like chirps) (Shumway, 1989a; Metzner and Juranek, 1997), may provide information about image amplitude that can be used to normalize the activity in the centromedial map, which is then used to compute image width and object distance. Normalization could be mediated by the extensive cerebellar-like feedback that projects to ELL, through shunting inhibition or synaptic depression (Maler and Mugnaini, 1994; Bastian, 1996; Berman and Maler, 1999). This simple hypothesis can be readily tested with established experimental techniques (Bastian, 1987; Metzner and Juranek, 1997; Nelson and MacIver, 1999). For example, ablating the lateral map of ELL (wide tuning) should disrupt the accurate estimation of image amplitude and the subsequent normalization step, resulting in an ambiguous estimation of object distance. So, predictable behavioral errors should occur when animals attempt 
to distinguish objects with certain combinations of size and distance.

The locus of computation of object distance is not known, and it need not be the centromedial map itself, because information from all ELL maps could be combined in higher brain regions (i.e., torus semicircularis or optic tectum) (Heiligenberg, 1991). Indeed, it is not necessary that there be a locus of computation, or explicit neural map, of object distance in electric fish. Such information could remain in a combined population code throughout its processing stream. However, there is some evidence of neurons in both the tectum and cerebellum that are tuned to object distance (Bastian, 1986a). Similar "distancetuned" neurons exist in the optic tectum of frogs and toads (House, 1989). There is also evidence that information from the different maps is treated very differently in the torus (Metzner and Juranek, 1997). So apparently the same information from different maps is not simply being averaged. Other constraints could lead to the formation of differently sized maps, such as specialized roles in temporal processing and communication (Metzner, 1999), as well as those proposed in the present paper.

The present analyses have primarily considered the location of an object. However, with an estimate of object distance $\left(\mathrm{z}^{*}\right)$, the approximate size of the object $\left(r_{o}\right)$ can then be decoded from the amplitude estimate (Eq. 3). The accuracy of this estimate will be constrained by the Cramer-Rao bound shown in Figure $3 a$. Thus, extensive cross-talk between ELL maps (either within ELL or in their projections to higher centers) may be required to identify the complete array of necessary object properties (Assad et al., 1999).

\section{Combined strategies in population coding}

The population coding literature has primarily dealt with how neuronal populations encode features to which its component neurons exhibit bell-shaped tuning curves. These studies often focus on how a single value of the feature in question can be extracted from the neuronal population response. There can be more information in the population response than that one value; for example, the entire probability distribution of a stimulus feature can be decoded from the population response (Zemel et al., 1998). There is recent evidence, in the case of visual motion perception, that such information is actually used to form a specific percept (Treue et al. 2000). This information is still related to the coarse-coded stimulus features. To our knowledge, extracting information from a combined population code in a functional context, has not been previously considered.

\section{Cues for electrosensory depth perception}

Our study of electrosensory depth perception has considered only static cues of object distance. In the context of visual processing, the problem is analogous to judging the depth of a stationary object using only monocular information. Electric images resulting from near objects are narrower and of greater peak amplitude than those of far objects, and thus can be considered as having less blur and higher contrast. Blur and contrast can have a significant influence on visual depth perception and are commonly used by artists in the pictorial depiction of depth (O'Shea et al., 1994; Mather, 1997). In normal visual processing, such cues are usually effective only in the absence of others such as those resulting from stereopsis and motion. Although there is no binocular analog in electrosensory processing, electric fish certainly have many motion cues available. Indeed, some species of electric fish exhibit a back-and-forth hovering motion that could be used to generate specific cues. Also, looming cues, such as those resulting from a changing electric image as an object approaches, could also be used for computing a parameter such as the time-tocollision, often discussed in the context of visual looming (Sun and Frost, 1998; Gabbiani et al., 1999; Rind and Simmons, 1999). As yet, there is little known about electrosensory motion processing and how electric fish might use such information for electrolocation.

\section{REFERENCES}

Abbott LF (1994) Decoding neuronal firing and modelling neural networks. Q Rev Biophys 27:291-331.

Abbott LF, Dayan P (1999) The effect of correlated variability on the accuracy of a population code. Neural Comput 11:91-101.

Assad C, Rasnow B, Stoddard PK (1999) Electric organ discharges and electric images during electrolocation. J Exp Biol 202:1185-1193.

Bastian J (1981) Electrolocation: II. The effects of moving objects and other electrical stimuli on the activities of two categories of posterior lateral line lobe cells in Apteronotus albifrons. J Comp Physiol 144:481-494.

Bastian J (1986a) Electrolocation: behavior, anatomy and physiology. Electroreception (Bullock TH, Heiligenberg W, eds), pp. 577-612. New York: Wiley.

Bastian J (1986b) Gain control in the electrosensory system: a role for the descending projections to the electrosensory lateral line lobe. J Comp Physiol [A] 158:505-515.

Bastian J (1987) Electrolocation in the presence of jamming signals: behavior. J Comp Physiol [A] 161:811-824.

Bastian J (1996) Plasticity in an electrosensory system. I. General features of a dynamic sensory filter. J Neurophysiol 76:2483-2496.

Berman NJ, Maler L (1999) Neural architecture of the electrosensory lateral line lobe: adaptations for coincidence detection, a sensory searchlight and frequency-dependent adaptive filtering. J Exp Biol 202:1243-1253.

Churchland PS, Sejnowski TJ (1992) The computational brain. Cambridge, MA: MIT

Deneve S, Latham PE, Pouget A (1999) Reading population codes: a neural implementation of ideal observers. Nat Neurosci 2:740-745.

Gabbiani F, Krapp HG, Laurent G (1999) Computation of object approach by a wide-field, motion-sensitive neuron. J Neurosci 19:1122-1141.

Heiligenberg W (1991) Neural nets in electric fish. Cambridge, MA: MIT.

House DH (1989) Depth perception in frogs and toads: a study in neural computing. New York: Springer.

Kay SM (1993) Fundamentals of statistical signal processing: estimation theory. New Jersey: Prentice Hall.

Khalsa PS, Friedman RM, Srinivasan MA, Lamotte RH (1998) Encoding of shape and orientation of objects indented into the monkey fingerpad by populations of slowly and rapidly adapting mechanoreceptors. J Neurophysiol 79:3238-3251.

Knudsen EI, du Lac S, Esterly SD (1987) Computational maps in the brain. Annu Rev Neurosci 10:41-65.

Konishi M (1986) Centrally synthesized maps of sensory space. Trends Neurosci 9:163-168.

Maler L, Mugnaini E (1994) Correlating gamma-aminobutyric acidergic circuits and sensory function in the electrosensory lateral line lobe of a gymnotiform fish. J Comp Neurol 345:224-252.

Mather G (1997) The use of image blur as a depth cue. Perception 26:1147-1158.

Metzner W (1999) Neural circuitry for communication and jamming avoidance in gymnotiform electric fish. J Exp Biol 202:1365-1375.

Metzner W, Juranek J (1997) A sensory brain map for each behavior? Proc Natl Acad Sci USA 94:14798-803.

Nelson ME, MacIver MA (1999) Prey capture in the weakly electric fish Apteronotus albifrons: sensory acquisition strategies and electrosensory consequences. J Exp Biol 202:1195-203.

O'Shea RP, Blackburn SG, Ono H (1994) Contrast as a depth cue. Vision Res 34:1595-604

Press WH, Teukolsky SA, Vetterling WT, Flannery BP (1993) Numerical recipes in $\mathrm{C}$ : the art of scientific computing, Ed 2. Cambridge: Cambridge UP.

Rasnow B (1996) The effects of simple objects on the electric field of Apteronotus. J Comp Physiol [A] 178:397-411.

Rind FC, Simmons PJ (1999) Seeing what is coming: building collisionsensitive neurones. Trends Neurosci 22:215-220.

Rosner B (1995) Fundamentals of biostatistics. Belmont, CA: Duxbury.

Salinas E, Abbott LF (1994) Vector reconstruction from firing rates. J Comput Neurosci 1:89-107. 
Shumway CA (1989a) Multiple electrosensory maps in the medulla of weakly electric gymnotiform fish. I. Physiological differences. J Neurosci 9:4388-4399.

Shumway CA (1989b) Multiple electrosensory maps in the medulla of weakly electric gymnotiform fish. II. Anatomical differences. J Neurosci 9:4400-4415.

Snippe HP, Koenderink JJ (1992) Discrimination thresholds for channel-coded systems. Biol Cybern 66:543-551.

Sun H, Frost BJ (1998) Computation of different optical variables of looming objects in pigeon nucleus rotundus neurons. Nat Neurosci 1:296-303.

Treue S, Hol K, Rauber HJ (2000) Seeing multiple directions of motionphysiology and psychophysics. Nat Neurosci 3:270-276.
Turner RW, Maler L (1999) Oscillatory and burst discharge in the apteronotid electrosensory lateral line lobe. J Exp Biol 202:1255-1265.

von der Emde G (1999) Active electrolocation of objects in weakly electric fish. J Exp Biol 202:1205-1215.

von der Emde G, Schwarz S, Gomez L, Budelli R, Grant K (1998) Electric fish measure distance in the dark. Nature 395:890-894.

Wheat H, Goodwin A, Browning A (1995) Tactile resolution: peripheral neural mechanisms underlying the human capacity to determine positions of objects contacting the fingerpad. J Neurosci 15:5582-5595.

Zemel RS, Dayan P, Pouget A (1998) Probabilistic interpretation of population codes. Neural Comput 10:403-430.

Zhang K, Sejnowski TJ (1999) Neuronal tuning: to sharpen or broaden? Neural Comput 11:75-84. 